fertem verhaftet, zuverlässig und unreflektiert Positionen in Justiz, Verwaltung

und Rechtsanwaltschaft auszuüben fähig sind.

Somit paßt in diese "Ausbildung", obwohl in $\$$ I JAO gefordert, nicht die Beschäftigung mit gesellschaftswissenschaftlichen Problemen und Erkenntnissen, wie insbesondere in Kategorie 2.6 nachgewiesen wurde. In der Stationsausbildung wie in den meisten Arbeitsgemeinschaften bilden diese Inhalte bestenfalls ein schmückendes Beiwerk mit Alibifunk tion.

Arbeitsgruppe Hamburger Gerchtsreferendare

\title{
Zur Post- und Telefonüberwachung in der BRD
}

\author{
Dr. Claus Arndt \\ $7 \mathrm{~g}-\mathrm{RF}-326$ \\ 205 Hamburg 80, am I 2. Mai 1974 [...]
}

An die Redaktion der Zeitschrift "Kritische Justiz« Sehr geehrte Damen und Herren!

Meinen Brief vom 12.1.1974 haben Sie in Ihrer Zeitschrift mit einem Kommentar Diethelm Damms abgedruckt, der seinerseits soviel Unrichtigkeiten enthält, daß eine Erwiderung unumgänglich erscheint:

Wie wenig orientiert Diethelm Damm über die Materie ist, über die er schreibt, zeigt mit entlarvender Deutlichkeit die Tatsache, daß Damm selbst für jedermann offenkundige und in allgemein zugänglichen Protokollen und Dokumenten enthaltene Fakten entweder nicht kennt oder verwechselt. so wundert er sich z. B., daß ich seit 1968 Mitglied der Dreierkommission nach $\$ 9$ Abs. 3 des Gesetzes zur Beschränkung des Brief-, Post- und Fernmeldegeheimnisses (Gesetz zu Art. IO GG) vom 1 3. 8. 1968 (BGBl. I S. 949) zu sein behauptet habe und bezweifelt insoweit die Zuver]ässigkeit meines Gedächtnisses. Er meint, die CDU/CSU habe noch am 2.7.1969 »die Einsetzung des ursprünglich geplanten Fünf-Männer-Gremiums... abgelehnt«. Mein Gedächtnis brauche ich dabei überhaupt nicht zu strapazieren: ich besitze noch heute meine erste Ernennungsurkunde, die ausweist, daß das Abgeordnetengremium nach $\$ 9$ Abs. 1 des Gesetzes zu Art. 10 GG mich in seiner Sitzung am 28. 1 I. 1968 nach vorhergegangener Anhörung der Bundesregierung zum Mitglied der Dreierkommission nach $\$ 9$ Abs. 3 des Gesetzes bestellt hat. Die Urkunde ist vom Bundestagsabgeordneten Dr. Bert Even, dem Vorsitzenden des Gremiums nach $\$ 9$ Abs. I, unterzeichnet. Die Dreierkommission hat sich noch am gleichen Tage konstituiert und dies in einem vom Bundestag verbreiteten amtlichen Pressekommunique unter namentlicher Nennung aller Mitglieder bekanntgemacht.

Am 2. 7. 1969 ging es um etwas ganz anderes, das mit Post- und Telefonkontrolle überhaupt nichts zu tun hatte. Wie jedermann in den Protokollen des Bundestages nachlesen kann (246. Sitzung, S. $13729 \mathrm{ff}$.), scheiterte an jenem Tage eine Grundgesetzänderung, durch die - unter mehren anderen Punkten das bis dahin seit mehreren Jahren aufgrund interner Vereinbarung zwischen allen Fraktionen des Bundestages und der Bundesregierung bestehende "Vertrauensmännergremium « des Bundestages in ein im Wortlaut der Verfassung ausdrücklich verankertes Verfassungsorgan umgewandelt werden sollte. Verantwortlich für das Scheitern war die CDU/CSU, deren Abgeordnete das Zustandekommen der Zweidrittelmehrheit im letzten Augenblick verhinderten, obwohl sie im Ausschuß zugestimmt hatten. Das Vertrauensmännergre- 
mium (das heute noch besteht) hatte und hat die Aufgabe, alle drei geheimen Dienste des Bundes parlamentarisch umfassend zu kontrollieren - seine Aufgabe geht daher zum Teil weiter und ist zum Teil enger als die Zuständigkeit des Abgeordnetengremiums nach $\$ 9$ Abs. I des Gesetzes zu Art. 10 GG. Jedenfalls handelt es sich um zwei vollkommen verschiedene Einrichtungen. Zumindest derjenige, der über Verfassungsschutzprobleme publiziert, sollte beide Institutionen nicht verwechseln.

Aber selbst bei der am 2.7. 1969 gescheiterten Verfassungsänderung ging es nicht darum, welche Bedeutung das Parlament der Kontrolle der Geheimdienste beimaß, sondern nur darum, ob diese Kontrolle effektiver sein könnte, wenn das Kontrollgremium, das immer bestand und arbeitete, ausdrücklich im Wortlaut des Grundgesetzes verankert ist (Bundestagsdrucksachen V/2425, $\mathrm{V} / 3965, \mathrm{~V} / 4445, \mathrm{~V} / 4514$ und zu V/4514). Diese Frage ist heute immer noch nicht entschieden und wird gegenwärtig unter meinem Vorsitz in der Unterkommission "Parlament und Regierung " der Enquetekommission des Bundestages für Fragen der Verfassungsreform erneut eingehend diskutiert.

Von ähnlich subtiler Sachkenntnis Diethelm Damms zeugen aber auch die anderen Behauptungen in seiner »Antwort« auf meinen Brief vom I 2. 1. 1974. Das gilt sowohl für die Technik der Telephonüberwachung wie für andere Fragen der Praxis der Postüberwachung. So könnten z. B. "Techniker des Verfassungsschutzes " Leitungen schon aus technischen Gründen gar nicht ohne Kenntnis und Mitwirkung von Postangehörigen umschalten, selbst wenn ihnen das nicht schon gesetzlich verboten wäre. Und die Feststellung, daß es wohl zwischen dem Bundesinnenminister, der eine Beschränkungsmaßnahme (Postoder Telephonüberwachung) anordnet, und dem Briefträger bzw. Postsortierer noch einige Instanzen bei der Post über die Oberpostdirektion und die Leitung des Postamts bis zum Stellenvorsteher gibt, die notwendigerweise mitwirken müssen, wird niemanden überraschen. Oder meint Damm, es gebe Briefträger, die entweder unmittelbar vom Innenminister oder von irgendeinem ihnen unbekannten »Verfassungsschützer« Anordnungen entgegennähmen, die die Behandlung der ihnen anvertrauten Postsendungen betreffen?

An Naivität kaum zu überbieten ist der Schluß Damms, weil niemand die Behauptung der Hanauer DKP dementiert habe, »in Stadt und Kreis Hanau würden I 230 Bürger telephonisch überwacht« (es dürfte gemeint sein, daß die Telephone der Bürger überwacht werden), sei dies eine Tatsache. Wenn die Oberwachung um der Sicherheit dieses Landes und seiner Bürger willen geheim erfolgt, dann kann man wohl kaum annehmen, diese Geheimhaltung ließe sich durch die Aufstellung irgendwelcher Behauptungen testen oder gar durchbrechen, indem man ein Dementi zu provozieren versucht oder aus seinem Ausbleiben Schlüsse zieht.

Damm ist zuzugeben, daß es bei der Post- und Telephonkontrolle Bereiche gibt und geben muß, bei deren Behandlung er auf Presseberichte angewiesen ist, deren Richtigkeit er wegen der notwendigen Geheimhaltung nicht immer nachprüfen kann. Um so sorgfältiger hätte Damm die relativ umfangreiche offen zugängliche Literatur und die jedermann einsehbaren amtlichen und nichtamtlichen Dokumente prüfen müssen. Daß dies nicht geschehen ist, bedingt, daß Damms Vermutungen und Behauptungen auch in anderen Punkten seiner "Antwort« ebenso wenig substantiiert sind wie bei der oben geschilderten Behandlung der verschiedenen Gremien. Das gilt ebenso hinsichtlich der Tätigkeit der Alliierten vor 1968 wie für Rechtslage und Praxis heute.

Und ein letztes Wort zum Stil einer Diskussion: ehe Damm mir unterstellt, ich 
hätte in meinem Brief vom I 2.1.1974 nicht nur Fakten ausgewählt, sondern auch solche unterschlagen, sollte er Roß und Reiter nennen und sagen, um welche Fakten es sich dabei handeln soll. Als Sozialist ist mir Diskussion Lebenselement. Mir hat noch keiner nachsagen können, ich hätte gekniffen. Ich gehe davon aus, daß Sie auch diesmal Ihre Leser teilhaben lassen und bin

mit freundlichen Grüßen: gez. Dr. Claus Arndt

\section{Bericht der Internationalen Juristenkommission über die Justiz in Chile*}

1. In Vertretung der Internationalen Juristenkommission besuchten wir Chile vom 19.-28. April 1974 mit dem Ziel, die juristische Situation in bezug auf die Menschenrechte zu untersuchen. Das Hauptziel unserer Mission war es, die gesetzlichen Garantien zu prüfen, die zur Zeit in Chile für die Personen, die aus politischen- und aus Sicherheitsgründen festgenommen sind, bestehen.

2. Wir hatten Besprechungen mit dem Innenminister, dem Justizminister, mit Mitgliedern des Obersten Gerichtshofs, der Rechtsanwaltskammer, mit dem Kardinal Raul Siva Henriquez und mit einer großen Anzahl von Personen, unter denen sich viele praktizierende Rechtsanwälte und Professoren der Rechtswissenschaft befanden. (...)

5. Chile wird zur Zeit nach dem Dekret, das der Verkündigung des »Ausnahmezustandes « und des »Kriegszustandes von Seiten der Militärjunta folgte, regiert. Nach diesen Verkündigungen sind alle demokratischen Rechte aufgehoben, einschließlich der Pressefreiheit, dem Recht zu Zusammenschlüssen und Zusammenkünften, der Meinungsfreiheit, den Gewerkschaftsrechten, dem Streikrecht und der Lehrfreiheit. Keine politische Tätigkeit ist erlaubt. Die politischen Parteien der vorherigen Regierungskoalition wurden für illegal erklärt und die anderen Parteien suspendiert. Eine nächtliche Ausgangssperre wurde verhängt.

(...)

7. Wenn man das herrschende Rechtssystem betrachtet, so stimmen wir der These zu, daß zur Zeit in Chile ein Ausnahmezustand herrscht, und wir haben versucht zu sehen, ob jene minimalen rechtlichen Garantien bestehen und jener Respekt vor den Rechten des Einzelnen, die auch in einer schweren Ausnahmesituation gegeben sein müssen. Insbesondere haben wir uns dafür interessiert, ob entsprechend den internationalen Verpflichtungen Chiles zur Zeit eines Bürgerkrieges (entsprechend dem Artikel 3 der Genfer Vereinbarungen von 1949) jene »juristischen Garantien, die als unabdingbar für zivilisierte Völker gelten, « aufrecht erhalten worden sind.

\footnotetext{
* Auszüge aus dem Vorläufigen Berıcht vom 17. 5. 1974. Ein abschließender Berıcht ist in Arbeıt. Zur Justız in Chile vgl. weiter:

- Dic eınem Teil der Auflage beıgelegte Dokumentatıon zum Prozeß gegen General Bachelet u. a. (Teile 1 und 2)

- Richter am Bundesgerıchtshof/Karlsruhe Woesner, Die Reste der Inquisitıon, In: Frankfurter Rundschau vom 8.6.1974, S. 2

- Liste der von der Militärjunta gefangenen und gefolterten Jurssten und Bediensteten der Justizverwaltung, in: „Chile-Nachrichten* Nr. I7/1974

Die Redaktıon der Chile-Nachrıchten bittet um Spenden zur Deckung der Druckkosten für die beiliegende Dokumentatıon über die gleıchfalls beiliegende Zahlkarte (Kennwort: Dokumentatıon).
} 